\title{
EXACT SEQUENCES IN STABLE HOMOTOPY PAIR THEORY
}

\author{
BY
}

K. A. HARDIE AND A. V. JANSEN ${ }^{1}$

\begin{abstract}
A cylinder-web diagram with associated diagonal sequences is described in stable homotopy pair theory. The diagram may be used to compute stable homotopy pair groups and also stable track groups of two-cell complexes. For the stable Hopf class $\eta$ the stable homotopy pair groups $G_{k}(\eta, \eta)(k \leqslant 8)$ are computed together with some of the additive structure of the stable homotopy ring of the complex projective plane.
\end{abstract}

0. Introduction. Let $\alpha \in G_{r}, \beta \in G_{m}$ denote stable classes of maps between spheres and let

$$
\pi_{k}=\operatorname{Dir}_{n \rightarrow \infty}\left[S^{n+k} \cup \cup_{\alpha} e^{n+r+k+1}, S^{n} \cup_{\beta} e^{n+m+1}\right]
$$

denote the corresponding stable track group. The groups $\pi_{r}$ have been studied by $\mathrm{N}$. Yamamoto [13] in the case $\alpha=\beta=p \neq 2$ and by J. Mukai [8, 9] in the case $\alpha=\beta=2$. In these papers rather extensive computations are given, but they use special information concerning the stable homotopy of Moore spaces. Here we develop a technique for computation which relies on the (stable) Puppe and dual-Puppe sequences passing through $\pi_{k}$ but which attempts to resolve the problems of group extension through the additional information contained in a cylinderweb diagram. Besides the rectangular mesh of Puppe and dual-Puppe sequences the cylinder-web diagram has three diagonal sequences (Theorem 3.3) that pass through the stable homotopy pair groups $G_{k}(\alpha, \beta)$. These groups are the natural "home" for (stable) Toda brackets of the form $\langle\beta, \gamma, \alpha\rangle$ in the sense that the brackets live here with zero indeterminacy.

In $\$ 6$ the technique is applied to compute, for the stable Hopf class $\eta$, the stable homotopy pair groups $G_{k}(\eta, \eta)(k \leqslant 8)$ and the stable track groups $\pi_{k}(k \leqslant 8)$ of the complex projective plane. It is interesting that besides knowledge of the stable homotopy groups of spheres, including composition and secondary composition operations, the method also requires information concerning the third order composition (quaternary Toda brackets). The computation presented as an illustration encountered a difficulty at $\pi_{9}$; however, it seems reasonable to expect that a better understanding of the quaternary bracket will enable it to be continued.

Received by the editors June 6, 1983 and, in revised form, December 7, 1983.

1980 Mathematics Subject Classification. Primary 55Q10; Secondary 55Q05.

${ }^{1}$ Grants to the Topology Research Group from the University of Cape Town and the South African Council for Scientific and Industrial Research are acknowledged. 
1. Preliminaries. Let $f: X \rightarrow Y, g: E \rightarrow B$ be pointed continuous maps (i.e. pairs in the sense of Eckmann and Hilton [1]). We recall that the morphism set $\pi(f, g)$ in the category HPC of homotopy pairs and homotopy pair classes $[3,4]$ is obtained from the set of tracks from $f$ to $g$ by factoring out by the equivalence relation:

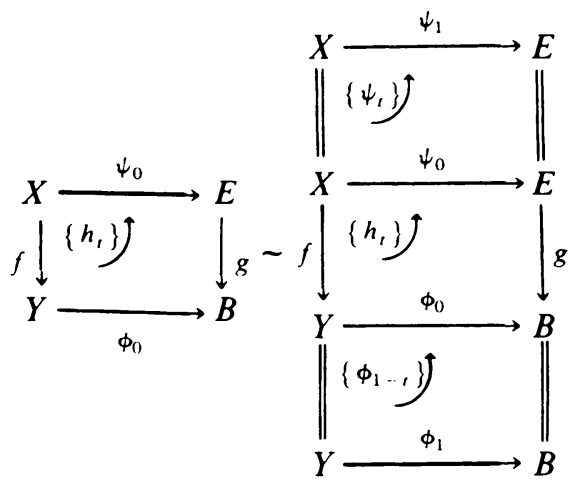

(The square referred to on the right is the composite in the obvious sense of the three internal squares.) The equivalence class of the element in $\pi(f, g)$ will be denoted $\left\{\phi, \psi, h_{t}\right\}$ unless $h_{t}$ is a constant homotopy in which case $\{\phi, \psi\}$ will be used.

If $X$ is a space, $* X, X *$ and $X$, respectively, will denote the inclusion of the basepoint $*$ into $X$, the projection of $X$ onto * and the identity map from $X$ to $X$. Similarly, it will be convenient to use the notation $X|f, * X| f, f \mid Y,(f, *)$ and $(*, f)$, respectively, for the commutative squares:
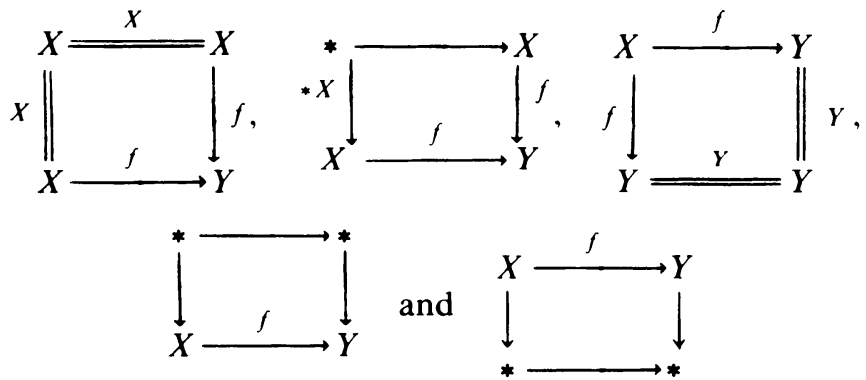

Following Puppe [10] we denote by $P f: Y \rightarrow C_{f}$ the inclusion of codomain $(f)$ into cofibre $(f)$. We recall from [4] that $P$ becomes an endofunctor of HPC if we set $P\left\{\phi, \psi, h_{t}\right\}=\{\chi, \phi\}$, where $\chi=C\left(\psi, \phi, h_{t}\right): C_{f} \rightarrow C_{g}$ is the map defined by the rule $[10,(9)]$. It was proved in [4] that $P^{3}$ is naturally isomorphic to $\Sigma$ in HPC and that the Puppe operator $P: \pi(f, g) \rightarrow \pi(P f, P g)$ eventually stabilizes. Let $Q f$ : $C_{f} \rightarrow \Sigma X$ be the map that shrinks to * the subset $Y$ of $C_{f}$ as described in [10, p. 308]. With morphisms defined as suggested [10, p. 312], $Q$ becomes an endofunctor of HPC, naturally isomorphic to $P^{2}$. We denote by $G_{k}(f, g)$ the stable group corresponding to $\pi\left(\Sigma^{k} f, g\right)$. As shown in [3], $\pi(f, g)$ is an invariant of the homotopy classes of $f$ and $g$. Hence $G_{k}(f, g)$ is an invariant of the stable homotopy classes of $f$ and $g$.

2. The stable cylinder-web diagram. Consider the following Diagram (2.1) of homotopy sets and induced functions. In the diagram, $f$ is the function induced by precomposition with the map $f$, and $g$. is the function induced by postcomposition 
with the map $g$. The horizontal sequences are all exact, being portions of the Puppe sequences of $f$ at $E, B$ or $C_{g}$. In the stable range, i.e. when $\Sigma$ is bijective, the vertical sequences are exact, being instances of (stable) dual-Puppe sequences of $g$. Since all horizontal arrows are induced by precomposition and all vertical arrows are induced by postcomposition, the rectangles not of the types indicated by (A), (B) and (C) are all commutative. Naturality of the Puppe sequence and naturality of the suspension operator ensures that the rectangles of type (C) are commutative.

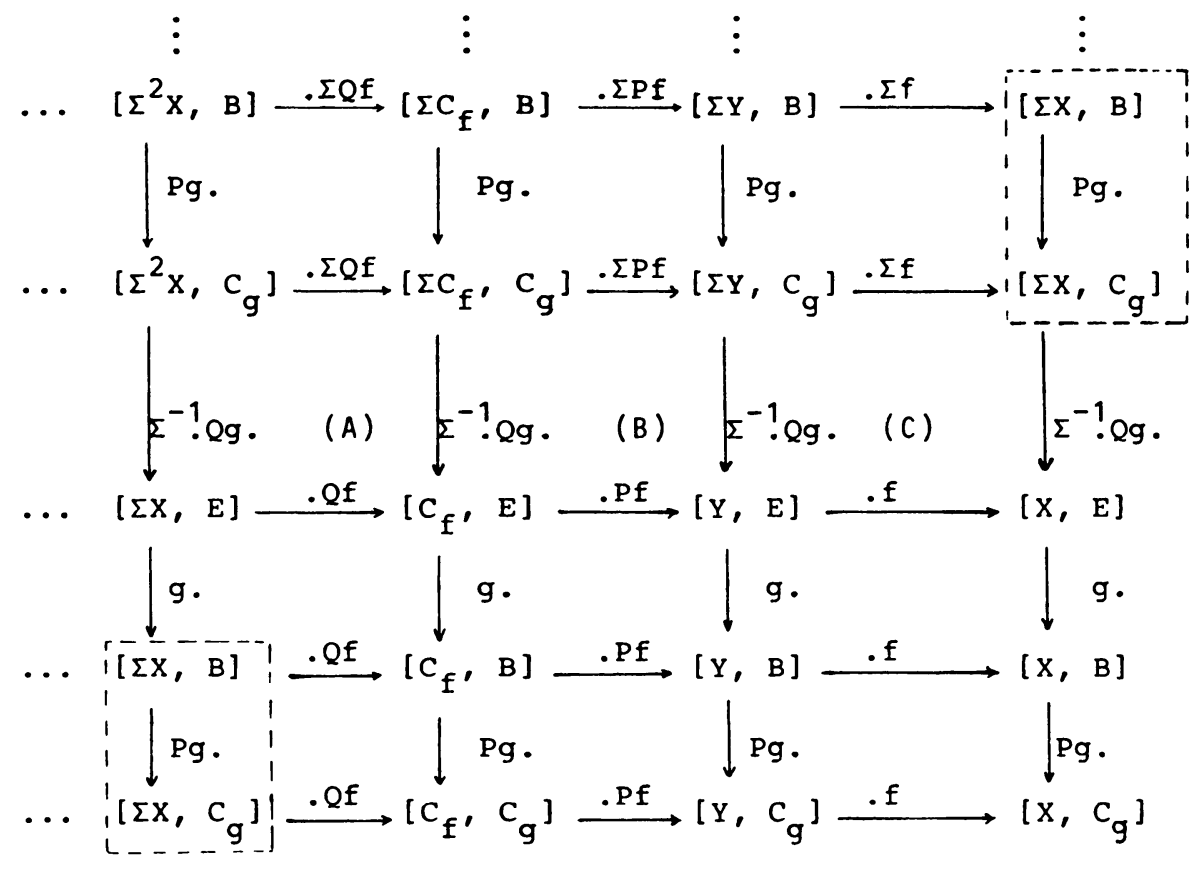

DiAgRAM 2.1

2.2. REMARK. There is a natural homeomorphism $\lambda: \Sigma C_{f} \rightarrow C_{\Sigma f}$ inducing a diagram (for each space $E$ )

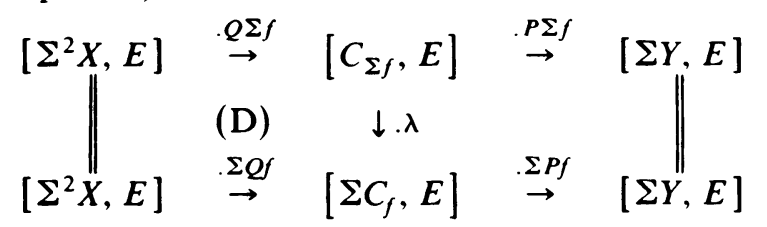

in which the right-hand square is commutative, but in which the left-hand square (D) is anticommutative through interchange of suspension coordinates in $\Sigma^{2} X$. Hence identifying via $\lambda$, the rectangles (B) are commutative and the rectangles (A) are anticommutative. We define

$$
\lambda_{n}: \Sigma^{n} C_{f} \rightarrow C_{\Sigma^{n} f} \text { by } \lambda_{1}=\lambda, \lambda_{n}=\lambda_{1}, \Sigma \lambda_{n-1} \text {. }
$$

One may now observe in Diagram 2.1 that objects and arrows separated by three diagonal shifts in a direction of positive slope are identical. (Two such arrows have been highlighted.) We have therefore another example of what has been described as a cylinder-web diagram $[2,3,5]$. The suspension operator provides a morphism of Diagram 2.1 into the corresponding diagram in which each arrow induced by a map 
is replaced by the arrow induced by the suspension of that map. Taking direct limit with respect to suspension yields the stable cylinder-web diagram.

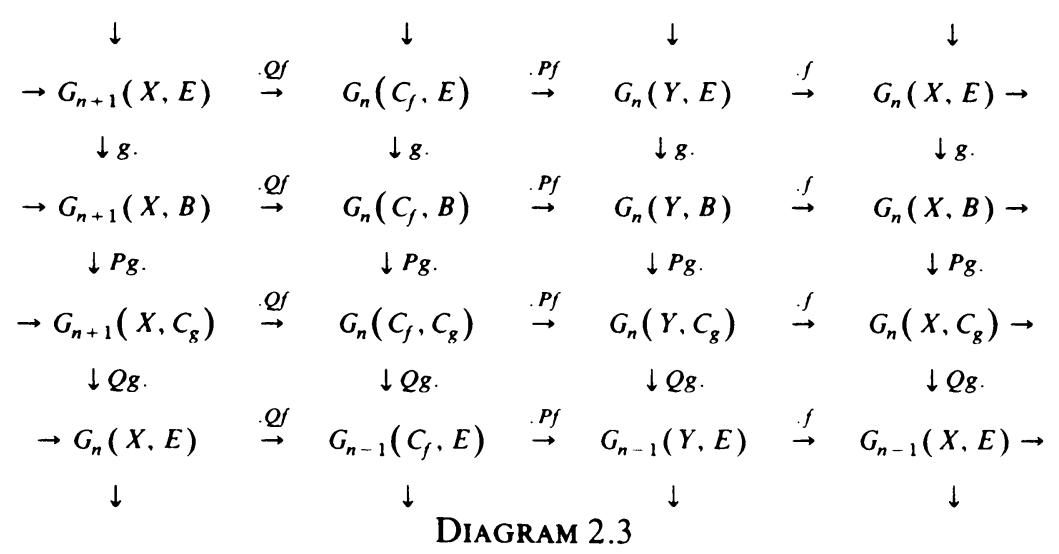

2.4. REMARK. The objects and arrows of 2.3 are invariants of the stable homotopy class of the map $f: X \rightarrow Y$. In the case of stable homotopy classes from $X$ to $Y$ which do not have representative maps from $X$ to $Y$, Diagram 2.3 is still obtained as a direct limit over a cofinal subset. Hence $f$ and $g$ may be permitted to be virtual maps.

3. The diagonal sequences. The domain and codomain operators $d: \pi(f, g) \rightarrow$ $[X, E]$ and $c: \pi(f, g) \rightarrow[Y, B]$ are defined by the rules $d\left\{\phi, \psi, h_{t}\right\}=\{\psi\}$ and $c\left\{\phi, \psi, h_{t}\right\}=\{\phi\}$. Recalling [4, §5] that $\Sigma\left\{\phi, \psi, h_{t}\right\}=\left\{\Sigma \phi, \Sigma \psi, \Sigma h_{t}\right\}$, it follows that $c$ and $d$ are compatible with $\Sigma$ and hence define stable operators $c: G_{n}(f, g) \rightarrow$ $G_{n}(X, E)$ and $d: G_{n}(f, g) \rightarrow G_{n}(Y, B)$. Further, recalling [5, §4] that the operator $\Delta=\Delta(f, g):[Y, E] \rightarrow \pi(f, g)$ is defined by the rule $\Delta\{h\}=\{g h, h f\}$, it is also clear that $\Delta$ is compatible with $\Sigma$, giving rise to an operator $\Delta: G_{n}(Y, E) \rightarrow G_{n}(f, g)$. Note that when the operators $P$ and $Q$ are bijective (as they are in the stable range [4]) the following composites are defined:

$$
\begin{gathered}
P^{-1} \Delta\left(P \Sigma^{n} f, P g\right)\left(. \lambda_{n}^{-1}\right):\left[\Sigma^{n} C_{f}, B\right] \rightarrow\left[\Sigma^{n} f, g\right], \\
Q^{-1} \Delta\left(Q \Sigma^{n} f, Q g\right)\left(. \lambda_{n}^{-1}\right):\left[\Sigma^{n+1} X, C_{g}\right] \rightarrow\left[\Sigma^{n} f, g\right] .
\end{gathered}
$$

In the course of the proof of Theorem 3.3 it will be shown that (3.1) and (3.2) are compatible with $\Sigma$ so that stable operators

$$
\Delta^{\prime}: G_{n}\left(C_{f}, B\right) \rightarrow G_{n}(f, g) \text { and } \Delta^{\prime \prime}: G_{n+1}\left(X, C_{g}\right) \rightarrow G_{n}(f, g)
$$

are also defined. A stable operator $\chi: G_{r}(f, g) \rightarrow G_{r}\left(C_{f}, C_{g}\right)$ is obtained if we set $\chi=\lambda_{n}^{-1} . . \lambda_{n} c P: \pi\left(\Sigma^{m} f, \Sigma^{n} g\right) \rightarrow\left[\Sigma^{m} C_{f}, \Sigma^{n} C_{g}\right]$.

To check the compatibility with $\Sigma$, observe commutativity in the following diagram:

$$
\begin{aligned}
& \pi\left(\Sigma^{m} f, \Sigma^{n} g\right) \longrightarrow \quad \pi\left(\Sigma^{m+1} f, \Sigma^{n+1} g\right) \\
& \downarrow c P \quad \text { (A) } \quad \downarrow c P \\
& {\left[C_{\Sigma^{m} f}, C_{\Sigma^{n} g}\right] \stackrel{\Sigma}{\rightarrow}\left[\Sigma C_{\Sigma^{m} f}, \Sigma C_{\Sigma^{n} g}\right] \stackrel{\lambda^{-1} . . \lambda}{\leftarrow} \quad\left[C_{\Sigma^{m+1} f}, C_{\Sigma^{n+1} g}\right]} \\
& \downarrow \lambda_{n}^{-1} . \lambda_{m} \quad \Sigma \lambda_{n}^{-1} . . \Sigma \lambda_{m} \longrightarrow \downarrow \lambda_{n+1}^{-1} \ldots \lambda_{m+1} \\
& {\left[\Sigma^{m} C_{f}, \Sigma^{n} C_{g}\right] \longrightarrow\left[\Sigma^{m+1} C_{f}, \Sigma^{n+1} C_{g}\right]}
\end{aligned}
$$


To verify the commutativity in (A), it is sufficient to consider $m=n=0$ and to verify the desired equality directly from the definition of the $P$ operator going back to [10, (9)].

Finally we shall need to refer to the diagonal composites

$$
\begin{aligned}
\beta & =g . . Q f: G_{n+1}(X, E) \rightarrow G_{n}\left(C_{f}, B\right), \\
\beta^{\prime} & =P g . . f: G_{n+1}(Y, B) \rightarrow G_{n+1}\left(X, C_{g}\right)
\end{aligned}
$$

and

$$
\beta^{\prime \prime}=Q g . . P f: G_{n+1}\left(C_{f}, C_{g}\right) \rightarrow G_{n}(Y, E) .
$$

3.3. THEOREM. The following sequences are exact:

$$
\begin{aligned}
& \cdots \rightarrow G_{n+1}(f, g) \stackrel{d}{\rightarrow} G_{n+1}(X, E) \stackrel{\beta}{\rightarrow} G_{n}\left(C_{f}, B\right) \stackrel{\stackrel{\Delta^{\prime}}{\rightarrow}}{\rightarrow} G_{n}(f, g) \rightarrow \cdots, \\
& \cdots \rightarrow G_{n+1}(f, g) \stackrel{c}{\rightarrow} G_{n+1}(Y, B) \stackrel{\beta^{\prime}}{\rightarrow} G_{n+1}\left(X, C_{g}\right) \stackrel{\Delta^{\prime \prime}}{\rightarrow} G_{n}(f, g) \rightarrow \cdots, \\
& \cdots \rightarrow G_{n+1}(f, g) \stackrel{x}{\rightarrow} G_{n+1}\left(C_{f}, C_{g}\right) \stackrel{\beta^{\prime \prime}}{\rightarrow} G_{n}(Y, E) \stackrel{\Delta}{\rightarrow} G_{n}(f, g) \rightarrow \cdots
\end{aligned}
$$

Proof. The sequence (3.4) is a stable version of the exact sequence $[5,4.3]$

$$
\cdots \rightarrow \pi\left(\Sigma^{n+1} f, g\right) \stackrel{d}{\rightarrow}\left[\Sigma^{n+1} X, E\right] \stackrel{\beta}{\rightarrow}\left[\Sigma^{n} C_{f}, B\right] \stackrel{\delta}{\rightarrow} \pi\left(\Sigma^{n} f, g\right) \rightarrow \cdots,
$$

where $\delta$ (for $n \geqslant 0$ ) is given by the following composition:

$$
\left[\Sigma^{n} C_{f}, B\right] \stackrel{\lambda_{n}}{\leftarrow}\left[C_{\Sigma^{n} f}, B\right] \stackrel{c P}{\leftarrow} \pi\left(\Sigma^{n} f, * B\right) \stackrel{* B \mid g .}{\rightarrow} \pi\left(\Sigma^{n} f, g\right)
$$

The argument for compatibility of $\delta$ with suspension is similar to that given above for the operator $\chi$. To check that $P^{-1} \Delta\left(P \Sigma^{n} f, P g\right)\left(. \lambda_{n}^{-1}\right)\{k\}=\delta\{k\}$, first observe that $\Delta(f, g)\{h\}=\{(E \mid g)(h, h)(f \mid Y)\}$, so that

$$
\begin{aligned}
P^{-1} \Delta\left(P \Sigma^{n} f, P g\right)\left(. \lambda_{n}^{-1}\right)\{k\} & =P^{-1}\left\{(B \mid P g)\left(k \lambda_{n}^{-1}, k \lambda_{n}^{-1}\right)\left(P \Sigma^{n} f \mid C_{\Sigma^{n} f}\right)\right\} \\
& =* B \mid g \cdot P^{-1}\left\{\left(k \lambda_{n}^{-1}, k \lambda_{n}^{-1}\right)\left(P \Sigma^{n} f \mid C_{\Sigma^{n} f}\right)\right\} .
\end{aligned}
$$

It is now sufficient to note that $\left(. \lambda_{n}\right) c\left\{\left(k \lambda_{n}^{-1}, k \lambda_{n}^{-1}\right)\left(P \sum^{n} f \mid C_{\Sigma^{n} f}\right)\right\}=\{k\}$.

The sequence (3.5) has essentially been given in $[4,8.1]$. However, this derivation is inconvenient for the purpose of recognition of the arrows. We sketch briefly an alternative. One may begin with the sequence

$$
\begin{gathered}
\cdots \rightarrow \pi\left(\left(\Sigma^{n} Y\right) *, * B\right) \stackrel{\cdot B \mid g \ldots\left(*, \Sigma^{n} f\right)}{\longrightarrow} \pi\left(\left(\Sigma^{n} X\right) *, g\right) \stackrel{\left.. \Sigma^{n} f K \Sigma^{n} X\right) *}{\longrightarrow} \\
\pi\left(\Sigma^{n} f, g\right) \stackrel{c}{\rightarrow}\left[\Sigma^{n} Y, B\right] \rightarrow \cdots
\end{gathered}
$$

The exactness can be verified directly, essentially dualising the proof of [5, Proposition 4.3]. In the form (3.7) the compatibility of the arrows with $\Sigma$ is obvious. The bijection

$$
\chi: \pi\left(\left(\Sigma^{n} Y\right) *, * B\right) \rightarrow\left[\Sigma^{n+1} Y, B\right]
$$


permits the sequence to continue, and, in the stable range,

$$
\chi: \pi\left(\left(\Sigma^{n} X\right) *, g\right) \rightarrow\left[\Sigma^{n+1} X, C_{g}\right]
$$

is a bijection, achieving recognition of one of the objects. It remains to verify the description of the arrow (3.2). Given $k: \Sigma^{n+1} X \rightarrow C_{g}$, we have

$$
\begin{aligned}
Q^{-1} \Delta\left(Q \Sigma^{n} f, Q g\right)\left(. \lambda_{n}^{-1}\right)\{k\} & =Q^{-1}\left\{\left(C_{g} \mid Q g\right)\left(k \lambda_{n}^{-1}, k \lambda_{n}^{-1}\right)\left(Q \Sigma^{n} f \mid \Sigma^{n+1} X\right)\right\} \\
& =\left(. \Sigma^{n} f \mid\left(\Sigma^{n} X\right) *\right) Q^{-1}\left\{\left(C_{g} \mid Q g\right)\left(k \lambda_{n}^{-1}, k \lambda_{n}^{-1}\right)\right\} .
\end{aligned}
$$

Since $d Q=c P$ it is sufficient to observe that $\left(. \lambda_{n}\right) d\left\{\left(C_{g} \mid Q g\right)\left(k \lambda_{n}^{-1}, k \lambda_{n}^{-1}\right)\right\}=\{k\}$.

The sequence (3.6) is a stable version of the "main diagonal" sequence $[5,4.7]$. However, the exactness is more easily established via a lemma on interlocking exact sequences [12, (1), p. 97]. Consider the following diagram in which sequences (3.4), (3.6) and two sequences from 2.3 interlock:

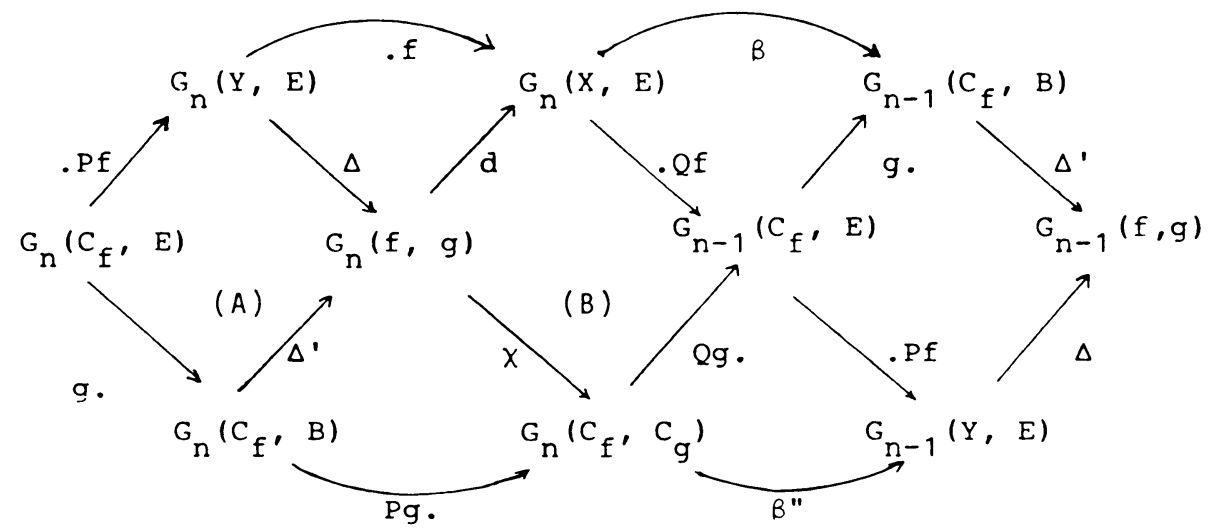

The commutativity of the triangles is easily checked and it is straightforward to verify that the rectangles (B) are commutative for $n$ even, and anticommutative for $n$ odd. To check the commutativity of the rectangles (A) let $h: \Sigma^{n} C_{f} \rightarrow E$. Then

$$
\Delta(. P f)\{h\}=\Delta\left(\Sigma^{n} f, g\right)\left\{h \Sigma^{n} P f\right\}=\left\{\left(h \Sigma^{n} P f \mid g\right)\left(\Sigma^{n} f \mid h \Sigma^{n} P f\right)\right\}
$$

and, since $\left(\Sigma^{n} P f\right)\left(\Sigma^{n} f\right)$ is nullhomotopic the homotopy pair class,

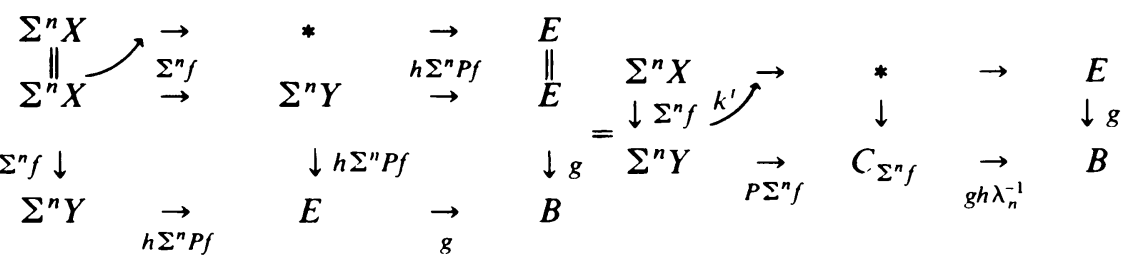

represents $\Delta(. P f)\{h\}$, where the homotopy $k_{t}$ can be chosen so that

$$
P\left(\begin{array}{llc}
\Sigma^{n} x & \vec{j} & * \\
\downarrow \Sigma^{n} f & k_{1} & \downarrow \\
\Sigma^{n} Y & \rightarrow & C_{\Sigma^{n} f}
\end{array}\right)=\left\{P \Sigma^{n} f \mid C_{\Sigma^{n} f}\right\} .
$$


Hence

$$
\begin{aligned}
P \Delta(. P f)\{h\} & =\left\{(B \mid P g)\left(g h \lambda_{n}^{-1}, g h \lambda_{n}^{-1}\right)\left(P \Sigma^{n} f \mid C_{\Sigma^{n} f}\right)\right\} \\
& =\Delta\left(P \Sigma^{n} f, P g\right)\left\{g h \lambda_{n}^{-1}\right\},
\end{aligned}
$$

establishing the desired equality. Since it is straightforward to check that (3.6) is differential at each object, an application of the lemma [12, (1), p. 97] completes the proof of Theorem 3.3.

There are many ways of displaying the cylinder-web diagram together with its diagonal sequences. The following conveniently separates the diagonal sequences (the labels of the horizontal and vertical arrows as shown in Diagram 2.3 have been suppressed):

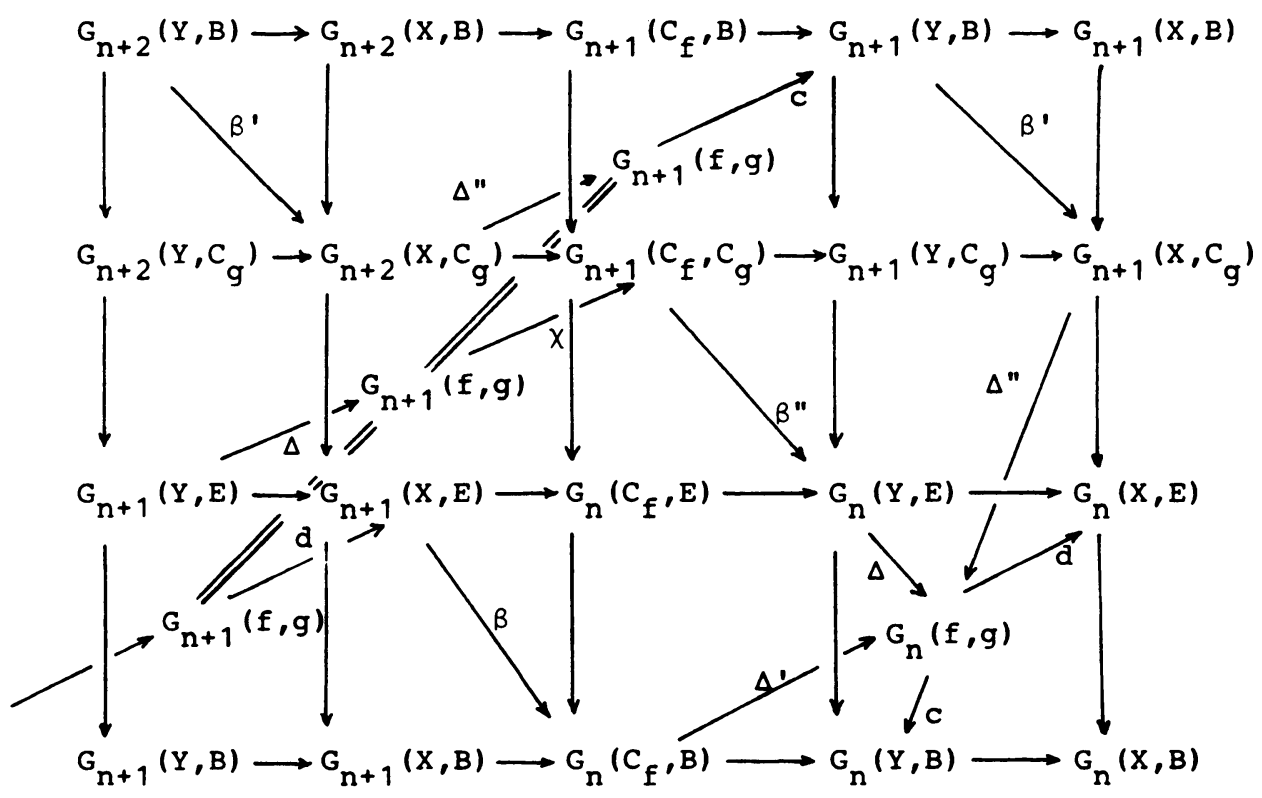

Diagram 3.9

It is intended that the relationship between the cylinder-web diagram and the diagrams of the type considered by C. T. C. Wall [12, p. 100] will be examined in a subsequent paper.

4. The stable Toda bracket. Let $f: X \rightarrow Y, h: \Sigma^{r} Y \rightarrow E, g: E \rightarrow B$ be maps, and suppose that, for some $n$, null-homotopies $m_{t}$ and $n_{t}$ of $\Sigma^{n}\left(h \Sigma^{r} f\right)$ and $\Sigma^{n}(g h)$ exist. The composite square

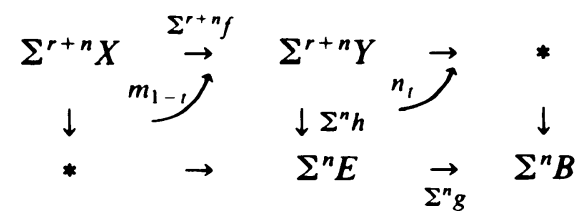


represents an element of $G_{r}(X *, * B)$, and applying $\chi$ we obtain an element of $G_{r+1}(X, B)$. The set of elements obtained in this way for all choices of $m_{t}$ and $n_{t}$ is denoted

$$
\langle\{g\},\{h\},\{f\}\rangle \subset G_{r+1}(X, B) .
$$

The definition is consistent with the definition of the stable Toda bracket as given in [7, p. 136].

4.3. TheOREM. Suppose that $\Sigma^{n}(g h) \simeq *$ and $\Sigma^{n}\left(h \Sigma^{r} f\right) \simeq *$. Then:

(a) for each element $\tau \in\langle\{g\},\{h\},\{f\}\rangle \subset G_{r+1}(X, B)$, we have

$$
\Delta^{\prime}(. Q f) \tau=-\Delta\{h\} \in G_{r}(f, g)
$$

(b) $\Delta\{h\}=0$ in $G_{r}(f, g)$ if and only if $0 \in\langle\{g\},\{h\},\{f\}\rangle$.

Proof. Let $\tau \in\langle\{g\},\{h\},\{f\}\rangle$. Then $-\tau$ has a representative of the form $\chi \alpha \in\left[\Sigma^{r+n+1} X, \Sigma^{n} B\right]$ where $\alpha$ is a composite square of the form:

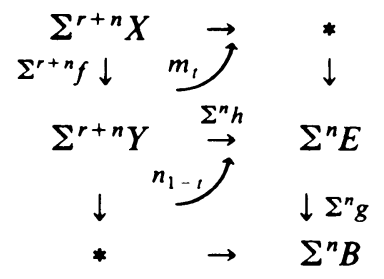

Hence $-\Delta^{\prime}(. Q f) \tau$ has a representative

$$
\begin{aligned}
\delta\left(. \Sigma^{r+n} Q f\right) \chi \alpha & =\delta \chi\left(\left(. \Sigma^{r+n} f \mid\left(\Sigma^{r+n} X\right) *\right) \alpha\right) \\
& =* \Sigma^{n} B\left|\Sigma^{n} g . . \Sigma^{r+n} f\right|\left(\Sigma^{r+n} X\right) *(\alpha)=\Delta\left\{\Sigma^{n} h\right\}
\end{aligned}
$$

the last step by the argument in the proof of [5, Theorem 5.3]. For part (b), observe that by $[12,(2)$, p. 97] commutativity of the diagram

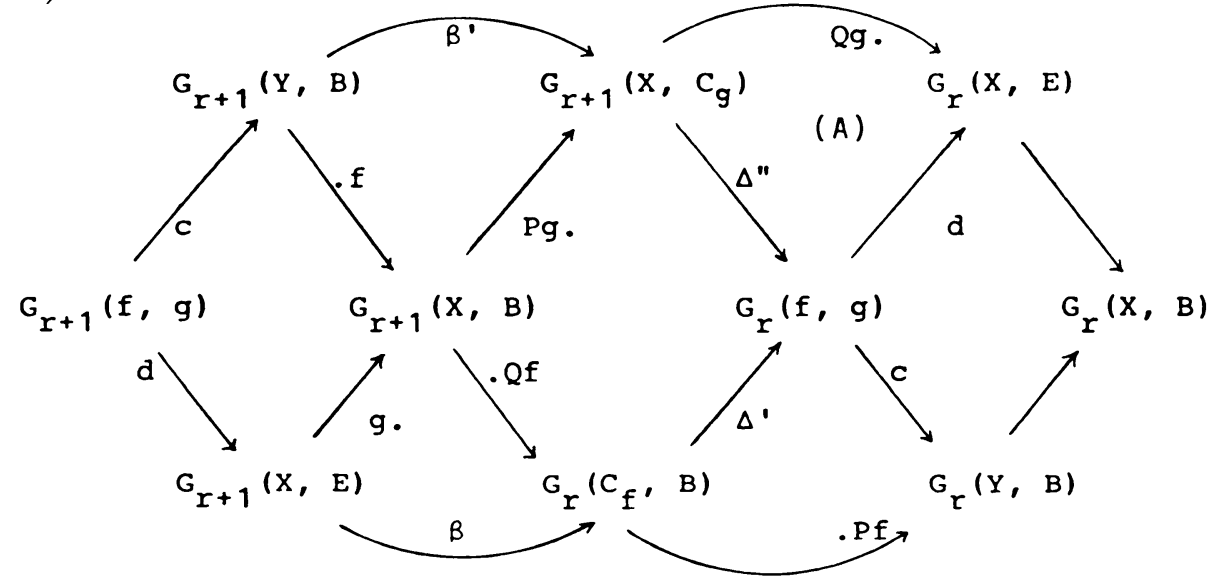

would yield a Mayer-Vietoris sequence

$$
\rightarrow G_{r+1}(f, g) \rightarrow G_{r+1}(X, E) \oplus G_{r+1}(Y, B) \rightarrow G_{r+1}(X, B) \stackrel{\Delta^{\prime}(. Q f)}{\rightarrow} G_{r}(f, g) \rightarrow
$$


in which the kernel of the arrow $\Delta^{\prime}(. Q f)$ is precisely the indeterminacy of $\langle\{g\},\{h\},\{f\}\rangle$. The reader may now easily check that the triangles (A) are commutative for $r$ even and anticommutative for $r$ odd, and further that the remaining triangles and rectangles of (4.4) are commutative.

Now let $\gamma=\{h\} \in G_{r}(Y, E)$ denote the stable homotopy class of $h$. The stable homotopy type of $C_{h}$ is an invariant of $\gamma$. Accordingly we shall denote by $C_{\gamma}$ a space $C_{h}$ for some $h$ in $\gamma$ and refer (with some abuse) to stable classes $P \gamma=\{P h\} \in$ $G_{0}\left(E, C_{\gamma}\right)$ and $Q \gamma=\{Q h\} \in G_{-r-1}\left(C_{\gamma}, Y\right)$. Moreover, if $\alpha=\{f\} \in G_{0}(X, Y)$ and $\beta=\{g\} \in G_{0}(E, B)$ are as above, we define

$$
\begin{gathered}
\text { Coext }_{\gamma} \alpha=\left\{\chi\left(\begin{array}{ccc}
\Sigma^{r+n} X & \overrightarrow{\Sigma^{r+n} f} & \Sigma^{r+n} Y \\
& \begin{array}{c}
m_{1-1} \\
*
\end{array} & \left.\mid \begin{array}{l}
\Sigma^{n} h \\
\Sigma^{n} E
\end{array}\right) \\
\text { null-homotopies } \\
m_{t} \text { of } \Sigma^{n}\left(h \Sigma^{r} f\right), \\
\text { for some } n
\end{array}\right\},\right. \\
\text { Ext }_{\gamma} \beta=\left\{\chi\left(\begin{array}{ccc}
\Sigma^{r+n} Y & \rightarrow & * \\
\downarrow \Sigma^{n} h & n_{t} & \downarrow \\
\Sigma^{n} E & \Sigma^{n} g & \Sigma^{n} B
\end{array}\right) \mid \begin{array}{l}
\text { null-homotopies } n_{t} \\
\text { of } \Sigma^{n}(g h), \text { for } \\
\text { some } n
\end{array}\right\} .
\end{gathered}
$$

We have the following

4.7. LeMMA. (i) $\langle\beta, \gamma, \alpha\rangle=\operatorname{Ext}_{\gamma} \beta \operatorname{Coext}_{\gamma} \alpha \subset G_{r+1}(X, B)$.

(ii) $\operatorname{Ext}_{\gamma} \beta=(. P \gamma)^{-1} \beta \subset G_{0}\left(C_{\gamma}, B\right)$.

(iii) $\operatorname{Coext}_{\gamma} \alpha=(Q \gamma .)^{-1} \alpha \subset G_{r+1}\left(X, C_{\gamma}\right)$.

Proof. Part (i) follows immediately from the definitions. Part (ii) is an easy consequence of the fact that $\chi\left(*\left(\Sigma^{n} E\right) \mid \Sigma^{n} h\right)=P \gamma$ and (iii) is a consequence of the equality $\left.\chi\left(\Sigma^{n} h\right)\left(\Sigma^{r+n} Y\right) *\right)=Q \gamma$.

Using the definition of (4.2) one may derive various standard properties of the stable Toda bracket. Particularly useful for the solution of group-extension problems that arise in computation is the following stable version of [11, Proposition 1.9]. A special case is given in [7, Lemma 1.2].

4.8. Proposition. If $\beta \gamma=0$ and $\gamma \alpha=0$ then $\left(\beta\right.$.) Ext $\operatorname{Ex}_{\alpha}=-(. Q \alpha)\langle\beta, \gamma, \alpha\rangle$ as $a$ coset of $(\beta).(. Q \alpha) G_{r+1}(X, E)$.

Proof. Since $\chi\left(\Sigma^{r+s} f\left(\Sigma^{r+s} X\right) *\right)=Q \alpha$, the desired result is a consequence of the following equality in HPC:

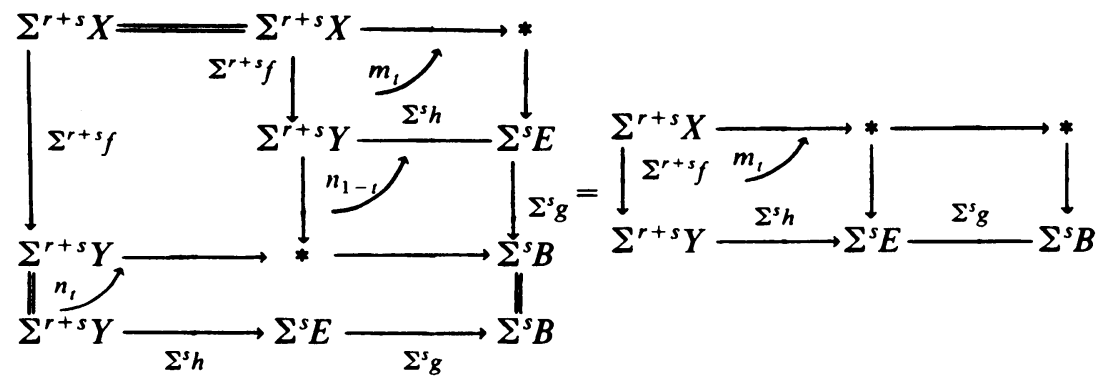

Proposition 4.8 is frequently used in the case $\beta=n$. 
4.9. CoROllaRy. If $n \gamma=0$ and $\gamma \alpha=0$ then $n \operatorname{Ext}_{\alpha} \gamma=-(. Q \alpha)\langle n, \gamma, \alpha\rangle$ as a coset of $n(. Q \alpha) G_{r+1}(X, E)$.

By a dual argument one may prove the following

4.10. Proposition. If $\beta \gamma=0$ and $n \gamma=0$ then $n \operatorname{Coext}_{\beta} \gamma=-(P \beta$. $)\langle\beta, \gamma, n\rangle$ as a coset of $n(P \beta$. $) G_{r+1}(Y, B)$.

5. The stable Puppe sequence of $\eta$. In $\$ \S 5$ and 6 the preceding theory will be applied to compute some stable homotopy of the cofibre of the stable Hopf class $\eta$. If we choose $f=g: S^{3}=X=E \rightarrow S^{2}=Y=B$ to be the Hopf map, then

$$
G_{r}\left(C_{f}, B\right)=\lim _{n \rightarrow \infty}\left[S^{r+n+2} \cup_{\eta} e^{r+n+4}, S^{n+2}\right]=\pi_{r+2}(\eta)
$$

and

$$
G_{r}\left(X, C_{g}\right)=\lim _{n \rightarrow \infty}\left[S^{r+n+3}, S^{n+2} \cup_{\eta} e^{n+4}\right]=\pi_{r+1}^{*}(\eta)
$$

in a standard notation [8]. The stable Puppe sequence of $\eta$ becomes as follows:

$$
G_{-1} \stackrel{. \eta}{\leftarrow} G_{-2} \stackrel{P}{\leftarrow} \pi_{0}(\eta) \stackrel{Q}{\leftarrow} G_{0} \stackrel{\cdot \eta}{\leftarrow} G_{-1} \stackrel{P}{\leftarrow} \pi_{1}(\eta) \stackrel{Q}{\leftarrow} G_{1} \stackrel{\eta}{\leftarrow} G_{0} \leftarrow \cdots .
$$

As essentially observed in [7, p. 141] the groups $\pi_{r}(\eta)(r \leqslant 22)$ can be counted. We use the notation for stable elements given in [11] and denote by ext $\alpha$ an element at random in the coset $\operatorname{Ext}_{\eta} \alpha$.

\subsection{Proposition.}

$\begin{array}{cccccc} & k=0 & k=1 & k=2 & k=3 & k=4 \\ \pi_{k}(\eta) \approx & Z & 0 & Z & Z_{4}+Z_{3} & 0 \\ \text { Generators } & Q & & \operatorname{ext} 2 & \nu Q \alpha_{1} Q & \\ & k=5 & k=6 & k=7 & k=8 \\ & Z_{8}+Z_{3} & Z_{2} & Z_{16}+Z_{3}+Z_{5} & Z_{4} \\ \operatorname{ext} \nu \operatorname{ext} \alpha_{1} & \nu^{2} Q & \sigma Q \alpha_{2} Q \alpha_{1} Q & \operatorname{ext} \nu^{2} \\ k=9 & k=10 & k=11 & k=12 \\ Z_{16}+Z_{3}+Z_{5} & Z_{3} & Z_{8}+Z_{2}+Z_{9}+Z_{7} & Z_{3} \\ \operatorname{ext} 2 \sigma \operatorname{ext} \alpha_{2} \operatorname{ext} \alpha_{1} & \beta_{1} Q & \operatorname{ext} \eta \varepsilon \operatorname{ext} \nu^{3} \alpha^{\prime} Q \alpha_{1} Q & \operatorname{ext} \beta_{1}\end{array}$

Proof. The computation via the Puppe sequence, given knowledge of the groups $G_{k}(k<19)$ as in [11], is quite straightforward except in cases where a problem of group extension arises. The first such case is $\pi_{8}(\eta)$. From the sequence

$$
\begin{array}{cr}
G_{8} \stackrel{. Q}{\longrightarrow} \pi_{8}(\eta) \stackrel{P}{\longrightarrow} G_{6} \\
Z_{2}+Z_{2} & Z_{2} \\
\bar{\nu} \varepsilon & \nu^{2}
\end{array}
$$

and knowing that $\eta \sigma=\bar{\nu}+\varepsilon$ and $\nu^{2} \eta=0$ [11] it follows that $\pi_{8}(\eta)$ is an extension of $Z_{2}$ by $Z_{2}$. It is claimed that $\varepsilon \in\left\langle 2, \nu^{2}, \eta\right\rangle$ from which Corollary 4.9 yields that $\varepsilon Q \in 2$ ext $\nu^{2}$, so that $\pi_{8}(\eta) \approx Z_{4}$, generated by ext $\nu^{2}$. To see that $\varepsilon \in\left\langle 2, \nu^{2}, \eta\right\rangle$ 
observe, firstly that $\varepsilon \in\left\langle\nu^{2}, 2, \eta\right\rangle[11$, p. 189] and, secondly, that the cosets $\left\langle\nu^{2}, 2, \eta\right\rangle,\langle\nu, 2 \nu, \eta\rangle,\langle 2 \nu, \nu, \eta\rangle$ and $\left\langle 2, \nu^{2}, \eta\right\rangle$ overlap and all have the same indeterminacy, namely $G_{7} \circ \eta$. The other cases in which extension problems arise are as follows:

$$
\begin{array}{rr}
G_{9} \longrightarrow \pi_{9}(\eta) \stackrel{. P}{\longrightarrow} G_{7} \\
\begin{array}{l}
Z_{2}+Z_{2}+Z_{2} \\
\nu^{3} \quad \mu \quad \eta \varepsilon
\end{array} & Z_{16}+Z_{3}+Z_{5} \\
\sigma & \sigma \alpha_{2} \alpha_{1}
\end{array}
$$

Here $\nu^{3} Q=\eta \varepsilon Q=0$ and $\pi_{9}(\eta)$ is an extension of $Z_{2}$ by $Z_{8}+Z_{3}+Z_{5}$. It is claimed that $\mu \in\langle 8,2 \sigma, \eta\rangle$ from which Corollary 4.9 yields $\mu Q \in 8$ Ext $2 \sigma$, settling the group extension. To see that $\mu \in\langle 8,2 \sigma, \eta\rangle$ observe that $\mu \in\langle 8 \sigma, 2, \eta\rangle$ [11, p. 189], and that $\langle 8 \sigma, 2, \eta\rangle \subset\langle 8,2 \sigma, \eta\rangle[7$, Proposition 1 (iii)]:

$$
\begin{array}{cc}
G_{11} \longrightarrow \pi_{11}(\eta) \stackrel{. P}{\longrightarrow} G_{9} \\
Z_{8}+Z_{9}+Z_{7} & Z_{2}+Z_{2}+Z_{2} \\
\zeta \alpha^{\prime} \alpha_{1} & \nu^{3} \mu \eta \varepsilon
\end{array}
$$

We have $\eta^{2} \mu=4 \zeta$ and $\nu^{3} \eta=\eta \varepsilon \eta=0$ so that $\pi_{11}(\eta)$ is an extension of $Z_{4}+Z_{9}+Z_{7}$ by $Z_{2}+Z_{2}$. Since $\zeta \in\langle 2, \eta \varepsilon, \eta\rangle[11$, p. 91, Lemma 9.1], we have $\zeta Q \in 2$ Ext $\eta \varepsilon$, so that $\pi_{11}(\eta) \approx Z_{9}+Z_{7}+Z_{8}+Z_{2}$, with the last two summands generated by ext $\eta \varepsilon$ and ext $\nu^{3}$, respectively.

5.2. Remark. The groups $\pi_{r}^{*}(\eta)$ may be computed through the dual Puppe sequence of $\boldsymbol{\eta}$ in a dual manner. Precisely the same extension problems arise and they are resolved via 4.10 so that $\pi_{r}(\eta) \approx \pi_{r}^{*}(\eta)$.

6. Computation of $\pi_{k}$. In this section the cylinder-web diagram together with 4.3 will be used to compute

$$
\pi_{k}=G_{k}\left(C_{\eta}, C_{\eta}\right)=\operatorname{Lim}_{n \rightarrow \infty}\left[S^{n+k} \cup_{\eta} e^{n+k+2}, S^{n} \cup e^{n+2}\right] \quad(k \leqslant 8)
$$

and, concurrently, $G_{k}(\eta, \eta)(k \leqslant 8)$. The diagram begins as indicated below (further to the right all the groups are trivial):

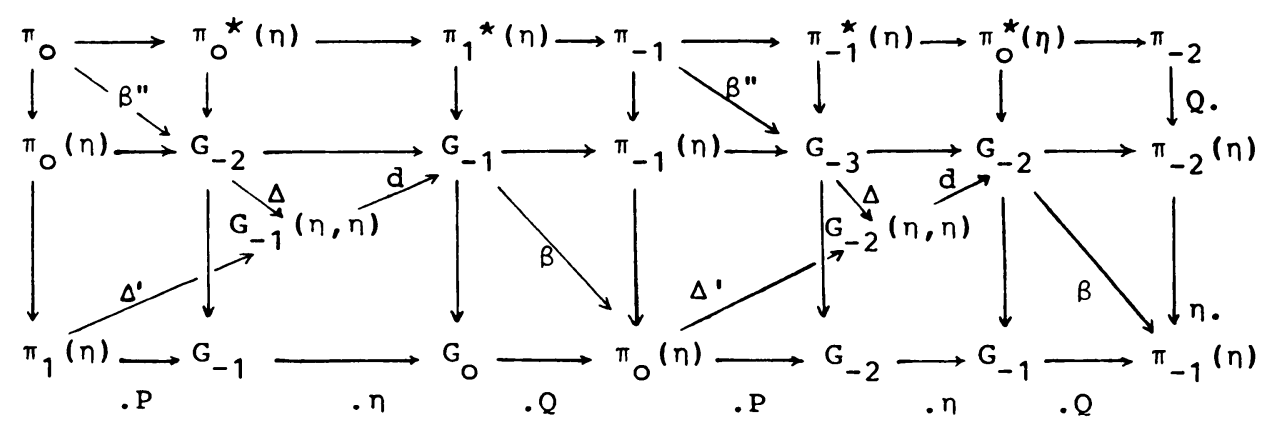

Note that all vertical arrows in the same row have the same label (either $\eta$. or $Q$.) and all horizontal arrows in the same column have the same label (namely . $P, Q$ or $. \eta)$. The diagonal sequence

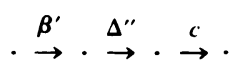


has not been shown and the arrows $\chi: G_{k}(\eta, \eta) \rightarrow \pi_{k}$ have also been suppressed. The symmetry of the diagram gives rise to a very desirable simplification:

\subsection{LEMMA. The arrows $\beta$ are all trivial.}

Proof. The commutation property $[11,(3.4)$, p. 33] implies that $\eta .= \pm . \eta$ hence $\beta=(. Q)(\eta)=. \pm(. Q)(. \eta)=0$.

As a consequence of 6.1 the diagonal $\Delta^{\prime}-d-\beta$ sequence becomes a collection of short exact sequences

$$
0 \rightarrow \pi_{k}(\eta) \stackrel{\Delta^{\prime}}{\rightarrow} G_{k-2}(\eta, \eta) \stackrel{d}{\rightarrow} G_{k-2} \rightarrow 0 .
$$

A similar (dual) argument shows that the arrows $\beta^{\prime}$ are all trivial so that embedded in the cylinder-web diagram are subdiagrams as follows:

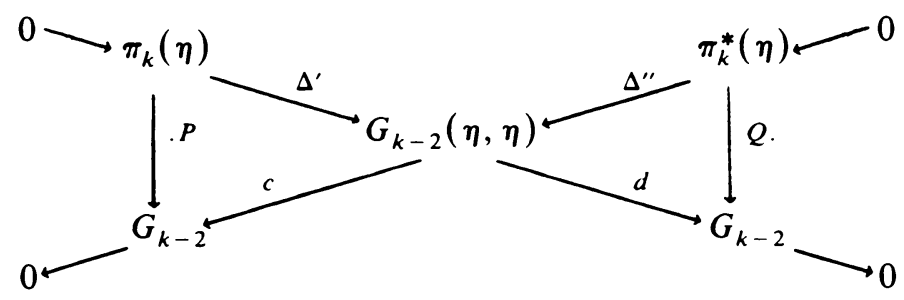

Clearly the short exact sequences split whenever .P or $Q$. is an isomorphism. From the nature of the nonsplit extensions it is also easy to see that a diagram

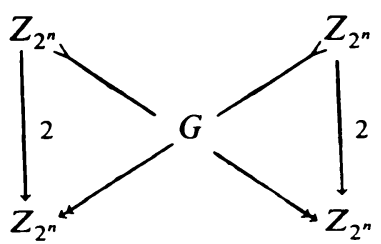

is only possible in the case $G \approx Z_{2^{n}}+Z_{2^{n}}$. The results of the computation are contained in the following theorems.

6.5. THEOREM.

$$
G_{k}(\eta, \eta)=\begin{array}{cccc}
k<-2 & k=-2 & k=-1 & k=0 \\
0 & Z & 0 & Z+Z \\
k=1 & k=2 & k=3 & k=4 \\
Z_{2}+Z_{4}+Z_{3} & Z_{2} & Z_{8}+Z_{8}+Z_{3}+Z_{3} & Z_{2} \\
k=5 & k=6 & k=7 & k=8 \\
Z_{16}+Z_{3}+Z_{5} & Z_{4}+Z_{2} & Z_{16}+Z_{16}+Z_{3}+Z_{3}+Z_{5}+Z_{5} & Z_{2}+Z_{2}+Z_{3}
\end{array}
$$

\subsection{THEOREM.}

$$
\pi_{k}=\begin{array}{cccc}
k<-2 & k=-2 & k=-1 & k=0 \\
0 & Z & 0 & Z+Z \\
k=1 & k=2 & k=3 & k=4 \\
Z_{2}+Z_{3} & Z & Z_{8}+Z_{4}+Z_{3}+Z_{3} & 0 \\
k=5 & k=6 & k=7 & k=8 \\
Z_{16}+Z_{4}+Z_{3}+Z_{3}+Z_{5} & Z_{4}+Z_{2} & Z_{16}+Z_{16}+Z_{3}+Z_{3}+Z_{5}+Z_{5} & Z_{4}+Z_{3}
\end{array}
$$


Proof of 6.5 AND 6.6. Since $\pi_{0}(\eta)=Z$, the sequence (6.3) with $k=0$ yields $G_{-2}(\eta, \eta)=0$, and the diagonal sequence $0=G_{-3} \rightarrow G_{-2}(\eta, \eta) \rightarrow \pi_{-2} \rightarrow 0$ implies $\pi_{-2}=Z$. Again, (6.3) with $k=1$ clearly yields $G_{-1}(\eta, \eta)=0$ and clearly $G_{-1}(\eta, \eta)$ $\approx \pi_{-1}$. With $k=2,(6.3)$ is $0 \rightarrow Z \rightarrow G_{0}(\eta, \eta) \rightarrow Z \rightarrow 0$, necessarily split. Moreover, $G_{0}(\eta, \eta) \approx \pi_{0}$ as in the previous case. With $k=3,(6.2)$ becomes $Z_{4}+Z_{3} \nrightarrow$ $G_{1}(\eta, \eta) \rightarrow Z_{2}$. As has been discussed in [5, Proposition 6.3], the extension is split and the image of $\Delta$ is an element of order 4 . It follows that $\pi_{1}$, the cokernel of $\Delta$, is isomorphic with $Z_{2}+Z_{3}$ [5, Corollary 6.4]. With $k=4,(6.2)$ becomes $0 \nrightarrow G_{2}(\eta, \eta)$ $\rightarrow Z_{2}$ and hence $G_{2}(\eta, \eta)=Z_{2}$. Moreover, $\Delta: G_{1} \rightarrow G_{2}(\eta, \eta)$ is surjective (since.$\eta$ : $G_{1} \rightarrow G_{2}$ factors through $\left.\Delta\right)$ and hence $\pi_{2}$ is isomorphic with the kernel of $\Delta$ : $G_{0} \rightarrow G_{1}(\eta, \eta)$ which is $Z$. With $k=5, . P: \pi_{5}(\eta) \rightarrow G_{3} \approx Z_{8}+Z_{3}$ is an isomorphism, and hence (6.3) implies (6.2) splits yielding $G_{3}(\eta, \eta)=Z_{8}+Z_{8}+Z_{3}+Z_{3}$. Since $\Delta: G_{2} \rightarrow G_{3}(\eta, \eta)$ is nontrivial, we have $\pi_{3}=$ cokernel $\Delta=Z_{8}+Z_{4}+Z_{3}+$ $Z_{3}$. With $k=6,(6.2)$ becomes $Z_{2} \nrightarrow G_{4}(\eta, \eta) \rightarrow 0$, hence $G_{4}(\eta, \eta)=Z_{2}$. Since $\nu^{2}=\langle\eta, \nu, \eta\rangle \in G_{6}, 4.3$ implies that $\Delta: G_{3} \rightarrow G_{4}(\eta, \eta)$ is surjective. Since also $\Delta$ : $G_{2} \rightarrow G_{3}(\eta, \eta)$ is nontrivial the exactness of the $\Delta-\chi-\beta^{\prime \prime}$ sequence implies that $\pi_{4}=0$. Since $G_{5}=0$, (6.2) with $k=7$ implies $G_{5}(\eta, \eta) \approx \pi_{7}(\eta)=Z_{16}+Z_{3}+Z_{5}$. The Puppe sequence passing through $\pi_{5}$ is

$$
\rightarrow \pi_{7}^{*}(\eta) \rightarrow \pi_{5} \rightarrow \pi_{5}^{*}(\eta) \rightarrow
$$

which raises the extension problem:

$$
\begin{aligned}
& 0 \rightarrow Z_{16}+Z_{3}+Z_{5} \quad \rightarrow \pi_{5} \rightarrow \quad Z_{4}+Z_{3} \quad \rightarrow 0 \\
& \begin{array}{lllll}
P \sigma & P \alpha_{2} & P \alpha_{1} & \operatorname{coext} 2 \nu & \operatorname{coext} \alpha_{1}
\end{array}
\end{aligned}
$$

Let $C_{\eta}$ denote the identity class of the cofibre of $\eta$.

6.7. LEMMA. $0 \in\left\langle 4 C_{\eta}\right.$, $\left.\operatorname{coext} 2 \nu, \eta\right\rangle \subset \pi_{7}^{*}(\eta)$.

Proof. Since $C_{\eta}=\operatorname{ext} P$ we have

$$
\left\langle 4 C_{\eta}, \operatorname{coext} 2 \nu, \eta\right\rangle=\langle\operatorname{ext} 4 P, \operatorname{coext} 2 \nu, \eta\rangle=\langle 4 P, \eta,-2 \nu, \eta\rangle,
$$

by the definition of the quaternary bracket [6, p. 174]. Since $\langle\eta, 2 \nu, \eta\rangle$ has zero indeterminacy and $0 \in\langle 2 \eta, \nu, \eta\rangle \subset\langle\eta, 2 \nu, \eta\rangle$, we have $\langle\eta, 2 \nu, \eta\rangle=0$. Also $0 \in$ $\langle 2 P, 2 \eta, 2 \nu\rangle$, which has the same indeterminacy as $\langle 4 P, \eta, 2 \nu\rangle$ so that $0 \in$ $\langle 2 P, 2 \eta, 2 \nu\rangle$. It follows that $\langle 4 P, \eta,-2 \nu, \eta\rangle$ is well defined. Moreover, we have

$$
0 \in\langle 4 P, \eta,-2, \nu \circ \eta\rangle \subset\langle 4 P, \eta,-2 \nu, \eta\rangle
$$

by [6, Proposition 2.9(ii)]. Lemma 6.7 implies that $0 \in 4 \operatorname{ext}(\operatorname{coext} 2 \nu$ ) so that $\pi_{5} \approx Z_{16}+Z_{4}+Z_{3}+Z_{3}+Z_{5}$. If $k=8$ then (6.3) becomes

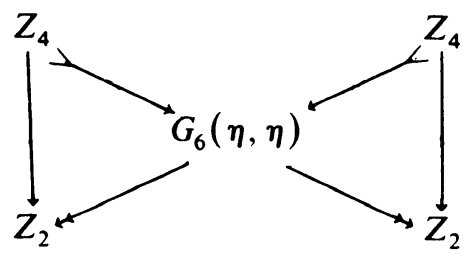


in which the vertical arrows are nontrivial. The split extension is the only one possible. Moreover, since $G_{4}=G_{5}=0$, we have that $\chi: G_{6}(\eta, \eta) \rightarrow \pi_{6}$ is an isomorphism. If $k=9$, the corner groups of (6.3) are each $Z_{16}+Z_{3}+Z_{5}$ and the 2-primary components yield an instance of (6.4). It follows that $G_{7}(\eta, \eta)=Z_{16}+$ $Z_{16}+Z_{3}+Z_{3}+Z_{5}+Z_{5}$. Since $0 \in\langle\eta, \nu, \nu \eta\rangle \subset\left\langle\eta, \nu^{2}, \eta\right\rangle$, an application of 4.3 yields that $\Delta: G_{6} \rightarrow G_{7}(\eta, \eta)$ is trivial, and since $G_{5}=0$ the $\Delta-\chi-\beta^{\prime \prime}$ sequence yields $\pi_{7} \approx G_{7}(\eta, \eta)$. Moreover, $\beta^{\prime \prime}: \pi_{8} \rightarrow G_{6}$ is epic from which it follows that $Q$ : $\pi_{8} \rightarrow \pi_{8}(\eta)=Z_{4}$ is epic. Since also $\pi_{10}(\eta)=Z_{3}=\left\{\beta_{1} Q\right\}$ and since $\eta .: \pi_{9}(\eta) \rightarrow$ $\pi_{10}(\eta)$ is trivial, we have $\pi_{8}=Z_{4}+Z_{3}$ and $G_{8}(\eta, \eta)=Z_{2}+Z_{2}+Z_{3}$. Attempting to determine $\pi_{9}$, we have $\pi_{11}^{*}(\eta)=Z_{8}+Z_{2}+Z_{9}+Z_{7}, \pi_{9}^{*}(\eta)=Z_{16}+Z_{3}+Z_{5}$ and a short exact sequence

$$
\pi_{11}^{*}(\eta) \nrightarrow \pi_{9} \rightarrow \pi_{9}^{*}(\eta) .
$$

Since the 2-primary component of $\pi_{9}^{*}(\eta)$ is generated by coext $2 \sigma$, the extension problem will be solved if we determine $\left\langle 16 C_{\eta}\right.$, coext $\left.2 \sigma, \eta\right\rangle \subset \pi_{11}^{*}(\eta)$. In this case the brackets $\langle 16 P, \eta, 2 \sigma\rangle$ and $\langle 16, \eta, 2 \sigma\rangle$ each have nontrivial indeterminacy so that in the sense of $[6$, p. 174] the quaternary bracket $\langle 16 P, \eta, 2 \sigma, \eta\rangle$ is not well defined. It is reasonable to expect that with a deeper understanding of the quaternary bracket this difficulty can be overcome.

\section{REFERENCES}

1. B. Eckmann and P. J. Hilton, Groupes d'homotopie et dualite, C. R. Acad. Sci. Paris Sér. I Math. 246 (1958), 2444, 2555, 2991

2. K. A. Hardie, Bifunctors and the diagonal exact sequences of a cylinder-web diagram, Quaestiones Math. 3 (1979), 169-179.

3. On the category of homotopy pairs. Topology Appl. 14 (1982), 59-69.

4. K. A. Hardie and A. V. Jansen, The Puppe and Nomura operators in the categon of homotop! '.s (Proc. Conf. on Categorical Aspects of Topology and Analysis, Carleton, 1980), Lectures Notes in $\lambda_{i}$, vol. 915, Springer-Verlag, Berlin and New York, 1982, pp. 112-126.

5. Toda brackets and the categon of homotopy pairs (Proc. Sympos. on Categorical Algebra and Topology, Cape Town, 1981), Quaestiones Math. 6 (1983), 107-128.

6. M. Mimura and H. Toda, On the generalized Hopf homomorphism and the higher composition, Part I, J. Math. Kyoto Univ. 4 (1964), 171-190.

7. M. Mori, Homotopy groups of Stiefel manifolds, Mem. Fac. Sci. Kyushu Univ. Ser. A 27 (1973), 135-148.

8. J. Mukai, Stable homotopy of some elementan complexes, Mem. Fac. Sci. Kyushu Univ. Ser. A 20 (1966), 266-282.

9. On the stable homotopy of a $Z_{2}$-Moore space, Osaka J. Math. 6 (1969), 63-91

10. D. Puppe. Homotopiemengen und ihre induzierten Abbildungen. I, Math. Z. 69 (1958), 299-344.

11. H. Toda, Composition methods in homotopy groups of spheres, Ann. of Math. Stud., vol. 49, Princeton Univ. Press, Princeton, N. J., 1962.

12. C. T. C. Wall, On the exactness of interlocking sequences, Enseign. Math. (2) 12 (1966), 95-100.

13. N. Yamamoto, Algebra of stable homotopy of Moore space. J. Math. Osaka City Univ. 14 (1963), $45-67$

Department of Mathematics, University of Cape Town, Rondebosch 7700, Republic of South AFRICA 